\title{
Curcumin promotes cell cycle arrest and apoptosis of acute myeloid leukemia cells by inactivating AKT
}

\author{
HAO ZHOU ${ }^{1,2}$, YICHONG NING ${ }^{3},{\text { GUIRONG } \text { ZENG }^{4}, \mathrm{CHANG} \mathrm{ZHOU}^{1} \text { and XIAOFENG DING }}^{2}$
}

\author{
${ }^{1}$ State Key Laboratory of Developmental Biology of Freshwater Fish, \\ ${ }^{2}$ The National and Local Joint Engineering Laboratory of Animal Peptide Drug Development, College of Life Sciences, \\ Hunan Normal University, Changsha, Hunan 410081; ${ }^{3}$ Department of Clinical Laboratory, Chongzuo People's Hospital, \\ Chongzuo, Guangxi 532200; ${ }^{4}$ Hunan Key Laboratory of Pharmacodynamics and Safety Evaluation of New Drugs and \\ Hunan Provincial Research Center for Safety Evaluation of Drugs, Changsha, Hunan 410331, P.R. China
}

Received September 29, 2020; Accepted January 19, 2021

DOI: 10.3892/or.2021.7962

\begin{abstract}
Curcumin, a phytochemical from rhizomes of the plant Curcuma longa, has been reported to exert potential anticancer properties in various cancer types, including acute myeloid leukemia (AML). However, the underlying mechanism remains poorly understood. The present study demonstrated that curcumin had a stronger cytotoxic activity against AML cells compared with three other types of phytochemicals (epigallocatechin gallate, genistein and resveratrol). Protein phosphorylation profiling using an antibody array identified that curcumin treatment increased the phosphorylation levels of 14 proteins and decreased those of four proteins. A protein-protein interaction network was constructed using the STRING database, in which AKT was identified as a hub protein with the highest connectivity (PRAS40, 4E-BP1, P70S6K, RAF-1 and p27). Western blotting results indicated that curcumin dose-dependently suppressed the phosphorylation of AKT, PRAS40, 4E-BP1, P70S6K, RAF-1 and p27 in AML cell lines (ML-2 and OCI-AML5). It was also demonstrated that curcumin regulated the cell cycle- and apoptosis-related proteins (cyclin D1, p21, Bcl2, cleaved-caspase-3 and cleaved-PARP), leading to cell cycle arrest and apoptosis in both ML-2 and OCI-AML5 cells. These effects of curcumin were enhanced
\end{abstract}

Correspondence to: Professor Chang Zhou, State Key Laboratory of Developmental Biology of Freshwater Fish, College of Life Science, Hunan Normal University, Changsha, Hunan 410081, P.R. China

E-mail: zhouc@hunnu.edu.cn

Professor Xiaofeng Ding, The National and Local Joint Engineering Laboratory of Animal Peptide Drug Development, College of Life Sciences, Hunan Normal University, Changsha, Hunan 410081, P.R. China

E-mail: dingxiaofeng@hunnu.edu.cn

Key words: acute myeloid leukemia, curcumin, AKT, cell cycle, apoptosis, afuresertib by the AKT inhibitor afuresertib but were suppressed by the AKT activator SC-79, indicating that curcumin functions via AKT. In the AML xenograft mouse model, curcumin and afuresertib synergistically suppressed the engraftment, proliferation and survival of AML cells. Collectively, the present study demonstrated that curcumin exerted anti-AML roles by inactivating AKT and these findings may aid in the treatment of AML.

\section{Introduction}

Acute myeloid leukemia (AML) is a hematological cancer type that is characterized by the clonal expansion and differentiation arrest of myeloid progenitor cells (1). The standard treatment for AML is induction chemotherapy, which is based on a backbone of cytarabine plus anthracycline treatment (2). However, the survival time for most patients who receive conventional therapy is short. Especially, the median survival of patients aged $\geq 65$ years is only 6 months (3). The 5 -year survival rate of patients with AML has not significantly increased despite significant advances in targeted therapy and immunotherapy over recent years (4). Therefore, there is an urgent requirement for the further identification of novel agents and therapeutics for AML.

Phytochemicals, which are natural compounds from plants, have been recognized as vital resources for novel drugs (5). For example, curcumin (6), epigallocatechin gallate (EGCG) (7), genistein (8) and resveratrol (9) have been reported to possess anti-AML properties. Curcumin is the main polyphenol component extracted from rhizomes of the plant Curcuma longa, and its therapeutic benefit has been demonstrated in various cancer types, including AML (10). However, the underlying mechanism is complex and remains poorly understood, as curcumin has multiple targets and is involved in various signaling pathways (11). Previous studies have reported that curcumin can exert its antitumor effects by acting as an inhibitor of kinases, such as protein kinase $\mathrm{B}(\mathrm{AKT} / \mathrm{PKB})$ in head and neck cancer cells (12), JAK1 in retinoblastoma cells (13) and p38MAPK in endothelial cells (14). In the present study, protein phosphorylation profiling using an antibody array demonstrated 
that curcumin treatment increased the phosphorylation levels of 14 proteins but decreased those of four proteins. Among the 18 proteins, AKT/PKB was found to be the main target of curcumin. Moreover, it was identified that curcumin promoted cell cycle arrest and apoptosis of AML cells by inactivating AKT.

\section{Materials and methods}

Chemicals and antibodies. Curcumin, genistein, epigallocatechin gallate (EGCG), resveratrol and decitabine were purchased from Target Molecule Corp. Afuresertib (GSK2110183) and SC79 were purchased from Selleck Chemicals. Antibodies against phosphorylated (p)-P70S6 kinase (P70S6K; T421/S424; cat. no. AP0540), p-AKT1(S473; cat. no. AP0140), total AKT1 (cat. no. A11016), poly(ADP-ribose) polymerase (PARP; cat. no. A11010), ACTB (cat. no. AF0198) and caspase 3 (cat. no. A2156) were obtained from ABclonal Biotech Co., Ltd. Antibodies against p-RAF-1 (S301; cat. no. AF0047), p-proline-rich Akt substrate, 40 kDa (PRAS40; T246; cat. no. AF2387), p-p27/Kip1 (T198; cat. no. AF3325), p-eukaryotic translation initiation factor 4E-binding protein 1 (4E-BP1; T36; cat. no. AF3431), $\beta$-tubulin (cat. no. AF7011) and Ki67 (cat. no. AF0198) were obtained from Affinity Biosciences. PE-conjugated (clone HI30; cat. no. 560975; BD Bioscience) and unconjugated mouse anti-human CD45 antibody (clone HI30; cat. no. 555480; BD Bioscience) were used for flow cytometry and immunohistochemistry (IHC), respectively. A FITC TUNEL cell apoptosis detection kit was purchased from Wuhan Servicebio Technology Co., Ltd.

Cell lines and culture. AML cell lines (HL-60, ML-2, MOLM-13, OCI-AML3, OCI-AML5 and U937) were obtained from the American Type Culture collection, and were cultured according to the manufacturer's instructions. All cell lines were mycoplasma-free and were authenticated by Yubo Biological Technology Co., Ltd. using short tandem repeat analysis.

Cytotoxicity assay. Cells were cultured in a 96-well plate until the cell confluence reached $\sim 70 \%$, and then cells were treated with different concentrations $(0,5,10,20,40$ and $80 \mu \mathrm{M})$ of curcumin, genistein, EGCG, resveratrol or decitabine. After $48 \mathrm{~h}$, cell viability was determined using a MTT assay as described previously (15). Based on the results of the MTT assay, the half maximal inhibitory concentration $\left(\mathrm{IC}_{50}\right)$ of each chemical was calculated.

Cell cycle and apoptosis analyses. As reviewed by Kouhpeikar et al (16), in vitro examination of the efficacy of curcumin against AML cells was conducted using 10-50 $\mu \mathrm{M}$ curcumin to treat cells for 24-48 h. In the present study, cells were treated with $25 \mu \mathrm{M}$ curcumin for $24 \mathrm{~h}$. After treatment, cell cycle and apoptosis were analyzed using a PI staining kit [Hangzhou Multi Sciences (Lianke) Biotech Co., Ltd.] and an Annexin V-FITC/PI staining kit (Invitrogen; Thermo Fisher Scientific, Inc.), respectively, according to the manufacturer's instructions. After staining, cells were analyzed using a flow cytometer (CytoFlex; Beckman Coulter, Inc.).
Phosphorylation profiling. A human phosphorylation pathway profiling array (cat. no. AAH-PPP-1-4) was purchased from RayBiotech, Inc., which can detect 55 phosphorylated proteins in five signaling pathways: MAPK, AKT, JAK/STAT, NF- $\mathrm{B}$ and TGF- $\beta$. ML-2 cells were cultured in a $10-\mathrm{cm}$ dish until cells reached $90 \%$ confluence, and then cells were treated with or without curcumin $(25 \mu \mathrm{M})$ for $6 \mathrm{~h}$. After treatment, the cells were harvested and lysed using the cell lysis buffer with a protease inhibitor cocktail and a phosphatase inhibitor cocktail. Phosphorylation array analysis was performed according to the manufacturer's protocol. The array was sequentially incubated with the sample and horseradish peroxidase-conjugated antibodies (provided within the kit), and then scanned with ImageQuant LAS4000 Scanner (Cytiva). In total, two biological replicates were performed, and the average expression levels were compared between the treatment and control samples.

Western blotting. Cells were cultured in a 12-well plate until the cell confluence reached $\sim 90 \%$, and then cells were treated with the indicated chemicals. Cell lysates were prepared using the cell lysis buffer with a protease inhibitor cocktail and a phosphatase inhibitor cocktail (Cell Signaling Technology). After quantification of the protein concentration, cell lysates containing equal amounts of total protein were denatured and separated on 10-12\% SDS-PAGE. Following separation, the proteins were blotted onto PVDF membranes and blocked. After sequentially incubated with primary antibodies and appropriate secondary antibodies, the membranes were exposed to Pierce ECL Western Blotting Substrate (Thermo Fisher Scientific, Inc.) and were imaged using a gel imaging system (Tanon 4600SF; Tanon Science and Technology Co., Ltd.).

Xenograft mouse models of AML. A total of 20 male NOD/SCID mice (age, 5-6 weeks; average weight, $23 \mathrm{mg}$ ) were purchased from Hunan Slaccas Jingda Laboratory Animal Co. Ltd., and housed in groups of 5 per cage with water and food ad libitum, in a specific-pathogen-free room with filtered air and controlled light/dark cycle $(12 / 12 \mathrm{~h})$, temperature $\left(24 \pm 2^{\circ} \mathrm{C}\right)$ and relative humidity $(45-65 \%)$. All mice were pretreated with an intraperitoneal injection of $20 \mathrm{mg} / \mathrm{kg}$ busulfan (APExBIO Technology LLC) $24 \mathrm{~h}$ before inoculation, and were then injected intravenously with $1 \times 10^{6}$ ML2 cells. At 15 days after inoculation, the mice were randomly divided into four groups ( 5 mice per group), and then treated with vehicle, curcumin ( $2 \mathrm{mg} / \mathrm{mouse})$, afuresertib (1 $\mathrm{mg} / \mathrm{mouse})$ or curcumin ( $2 \mathrm{mg} / \mathrm{mouse})+$ afuresertib (1 mg/mouse) via oral gavage every other day for 16 days. Curcumin and afuresertib were dissolved with $5 \%$ DMSO $+10 \%$ PEG300 + 5\% Tween-80. The humane endpoints were defined by body weight loss of $20 \%$. All mice were euthanized by asphyxiation $\left(\mathrm{CO}_{2}\right.$ displacement rate was $\sim 20 \% \mathrm{vol} / \mathrm{min}$ ) 4 days after the last treatment, and the death was verified by respiratory arrest and cardiac arrest for $>10 \mathrm{~min}$. The experiments were performed from to July 10 to August 12. The spleens were fixed in $10 \%$ formalin and processed for hematoxylin and eosin (H\&E) staining, immunohistochemistry (IHC) analysis and TUNEL assay, as described previously $(17,18)$. Bone marrow (obtained from tibias and femurs) was crushed 
Table I. $\mathrm{IC}_{50}$ values (mean $\pm \mathrm{SD}$ ) of four phytochemicals and decitabine/curcumin against AML cell lines.

\begin{tabular}{lccccc}
\hline Cell line & Decitabine & Curcumin & EGCG & Genistein & Resveratrol \\
\hline HL-60 & $69.13 \pm 13.65$ & $46.98 \pm 0.79$ & $111.94 \pm 34.72^{\mathrm{d}}$ & $90.24 \pm 15.65$ & $60.55 \pm 3.67$ \\
ML-2 & $33.67 \pm 1.57^{\mathrm{d}}$ & $21.51 \pm 0.46^{\mathrm{b}}$ & $34.65 \pm 1.81^{\mathrm{d}}$ & $40.10 \pm 2.13^{\mathrm{b}, \mathrm{d}}$ & $28.70 \pm 1.29^{\mathrm{a}, \mathrm{d}}$ \\
MOLM-13 & $54.02 \pm 11.89$ & $53.18 \pm 5.87$ & $102.11 \pm 2.77^{\mathrm{b}, \mathrm{d}}$ & $59.65 \pm 10.02$ & $64.93 \pm 5.01$ \\
OCI-AML3 & $55.39 \pm 8.37$ & $71.43 \pm 10.12$ & $78.65 \pm 18.07$ & $100.01 \pm 20.15^{\mathrm{a}}$ & $48.01 \pm 8.11$ \\
OCI-AML5 & $126.76 \pm 8.54^{\mathrm{d}}$ & $38.45 \pm 0.38^{\mathrm{b}}$ & $120.17 \pm 21.37^{\mathrm{d}}$ & $64.59 \pm 2.13^{\mathrm{b}, \mathrm{d}}$ & $70.26 \pm 0.32^{\mathrm{b}, \mathrm{c}}$ \\
U937 & $56.10 \pm 2.17$ & $59.80 \pm 1.34$ & $74.44 \pm 1.40^{\mathrm{b}, \mathrm{d}}$ & $95.32 \pm 5.40^{\mathrm{b}, \mathrm{d}}$ & $74.32 \pm 4.20^{\mathrm{b}, \mathrm{d}}$
\end{tabular}

Differences between each phytochemical and decitabine (curcumin) were analyzed by Tukey test; adjust $\mathrm{P} \leq 0.05$ and ${ }^{\mathrm{b}}$ adjust $\mathrm{P} \leq 0.01$ vs. decitabine; cadjust $\mathrm{P} \leq 0.05$ and ${ }^{\mathrm{d}}$ adjust $\mathrm{P} \leq 0.01$ vs. curcumin. $\mathrm{IC}_{50}$, half maximal inhibitory concentration; AML, acute myeloid leukemia; EGCG, epigallocatechin gallate.

in PBS and created into single cell suspensions for flow cytometry analysis.

Statistical analysis. RStudio (https://rstudio.com) was used for statistical analysis. ANOVA and Tukey's post hoc test were performed to evaluate the significance of difference between samples, adjust $\mathrm{P}<0.05$ was considered as the level of significance.

\section{Results}

Screening for anti-AML phytochemicals. The four phytochemicals (curcumin, EGCG, genistein and resveratrol) have been reported to function as epigenetic modulating agents in cancer (19), while the DNA methyltransferase inhibitor decitabine is an FDA-approved chemical for the treatment for AML. Thus, the present study compared the cytotoxicity of these four phytochemicals with decitabine in six AML cell lines (HL-60, ML-2, MOLM-13, OCI-AML3, OCI-AML5 and U937). Cell viabilities at $48 \mathrm{~h}$ after exposure to various concentrations of drugs were determined using MTT assays, and $\mathrm{IC}_{50}$ values were calculated. Compared with decitabine, curcumin had a similar or lower $\mathrm{IC}_{50}$ in the $\mathrm{AML}$ cell lines (Table I). Moreover, curcumin had the strongest cytotoxic activity against AML cells (except for OCI-AML3) among the four phytochemicals, and ML-2 cells were the most sensitive to curcumin. Therefore, curcumin was selected for further study of its function and mechanism in AML.

Phosphorylation array analysis indicates AKT is the key target of curcumin. It has been reported that curcumin can affect protein phosphorylation (12-14). Thus, the influence of curcumin treatment on protein phosphorylation was examined using a human phosphorylation pathway profiling array, which can detect 55 phosphorylated proteins in five signaling pathways: MAPK, AKT, JAK/STAT, NF- $\mathrm{B}$ and TGF- $\beta$. The results demonstrated that the phosphorylation levels of 14 proteins were downregulated (fold change $\leq 0.83$ ), while those of four proteins were upregulated (fold change $\geq 1.2$ ) after treatment with curcumin for $6 \mathrm{~h}$ (Fig. 1A; Table SI). Functional annotation analysis based on the Kyoto Encyclopedia of Genes and Genomes (KEGG) database identified that the five most enriched pathway were the 'PD-L1/L1 pathway',
'AML', 'ErbB signaling pathway', 'EGFR tyrosine kinase inhibitor (TKI) resistance' and 'B cell receptor signaling pathway' (Fig. 1B; Table SII).

To understand the association between these 18 proteins, a protein-protein interaction (PPI) network was constructed using STRING software (https://string-db.org). In a PPI network, the nodes (proteins) with multiple edges (interactions) are defined as hubs, and hubs are more essential for the global network structure compared with non-hubs (20). In the present network (Fig. 1C), the AKT node had the highest number of edges, indicating that AKT was the key target of curcumin. Moreover, AKT was involved in the top 20 pathways affected by curcumin (Table SII).

To confirm the result of the phosphorylation array analysis, western blotting was conducted to detect the influence of curcumin (CCM) on the phosphorylation status of AKT and its interacting proteins in ML-2 cells. Curcumin suppressed the phosphorylation of AKT1, PRAS40, 4E-BP1, P70S6K, RAF-1 and p27 in a dose-dependent manner (Fig. 1D).

Curcumin promotes $A M L$ cell arrest in the $G_{1}$ phase by inactivating $A K T$. To determine whether the cytotoxicity of curcumin is dependent on AKT activity, AML cells were treated with curcumin (CCM), an AKT activator (SC-79) or an AKT inhibitor (afuresertib) alone (AFU) or in combination $(\mathrm{CCM}+\mathrm{SC} 79 / \mathrm{CCM}+\mathrm{AFU})$. Based on the results of the MTT assay (Fig. 2A), SC-79 reversed the antitumor effects of curcumin, while afuresertib augmented its cytotoxicity on both OCI-AML5 and ML-2 cells. Flow cytometry results demonstrated that treatment with curcumin or the AKT inhibitor afuresertib led to cell cycle arrest in the $G_{1}$ phase, while treatment with the AKT activator SC-79 promoted cell division (Fig. 2B and C). Moreover, AKT activation by SC-79 rescued the curcumin-induced cell cycle arrest, while AKT inhibition by afuresertib enhanced this effect (Fig. 2B and C). These results suggested that curcumin suppressed AML cell arrest in the $\mathrm{G}_{1}$ phase by inactivating AKT.

The influence of curcumin, SC-79 and afuresertib on cyclin D1 and p21, which are positive and negative regulators of the cell cycle progression from the $G_{1}$ to $S$ phase $(21,22)$, respectively, was then examined. Treatment of the AML cell lines with curcumin or afuresertib suppressed the expression of cyclin D1, but increased the expression of p21 (Fig. 2D and E). 
A
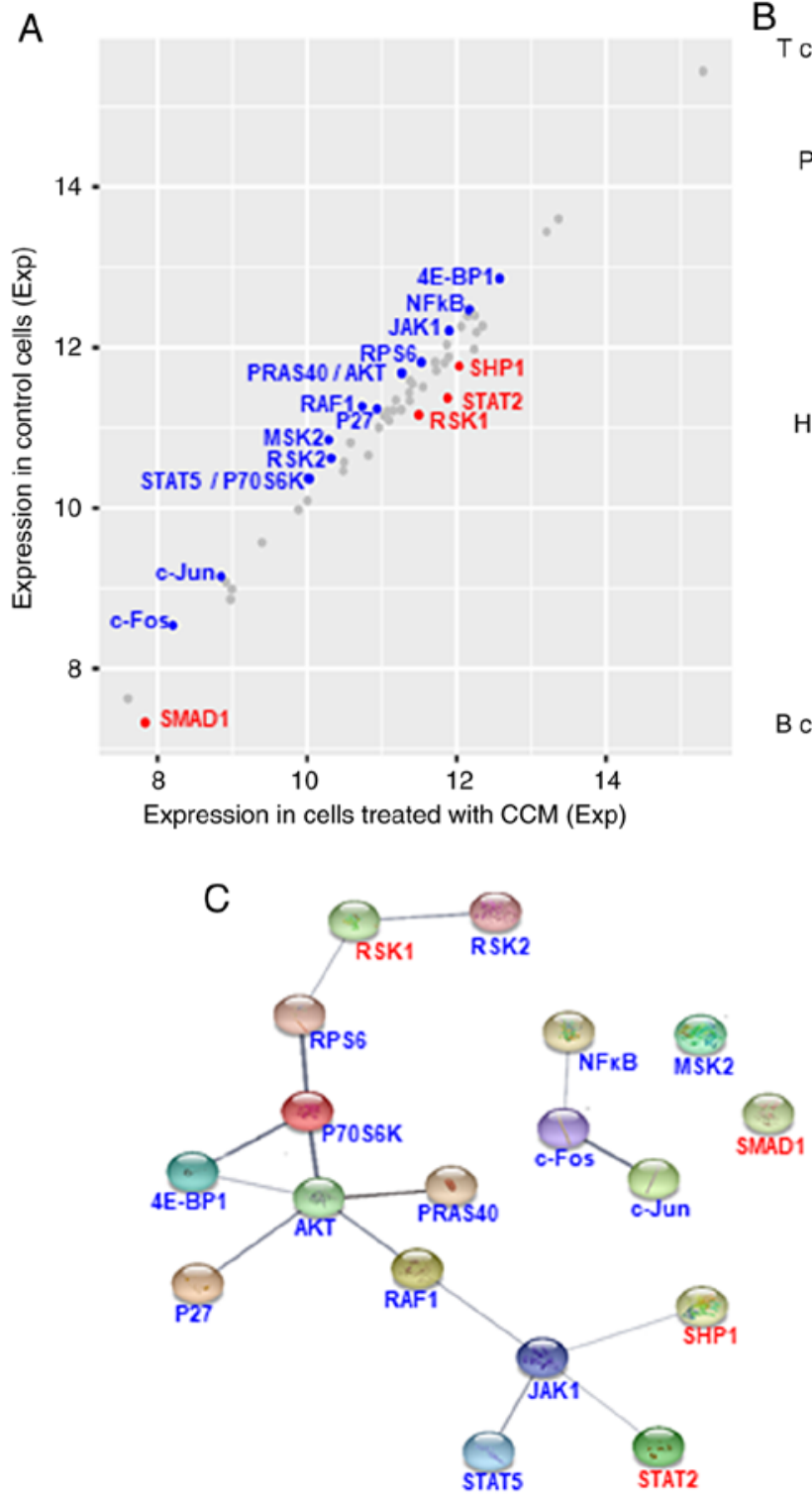

B
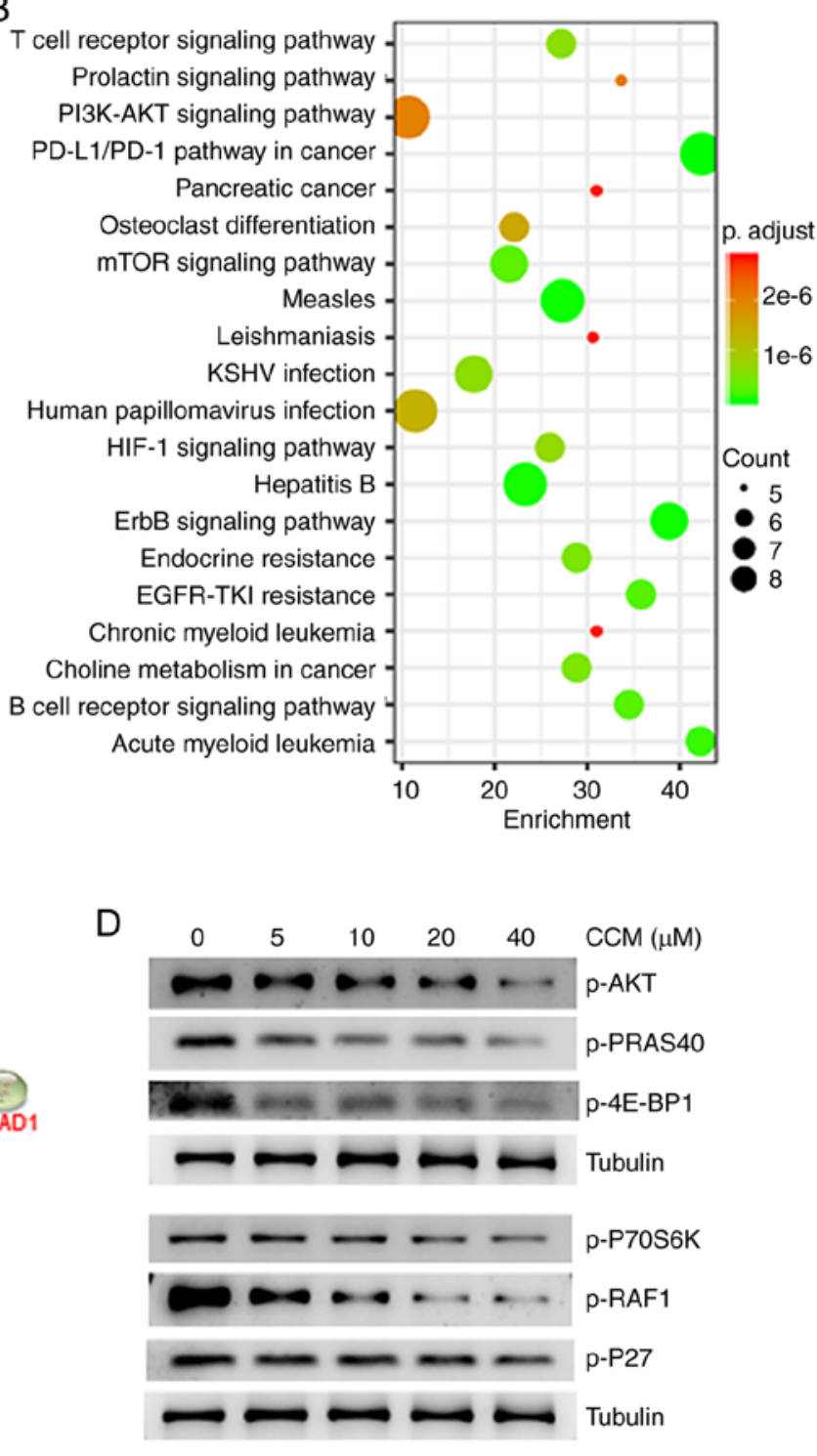

Figure 1. Influence of curcumin (CCM) on protein phosphorylation, as detected via antibody array and western blotting. (A) Scatter plot demonstrating the relative expression levels of the 55 phosphorylated proteins. ML-2 cells were treated with or without CCM for 6 h. After treatment, a phosphorylation antibody array was used for detecting the relative levels of phosphorylation. The red dots represent upregulated proteins, the blue dots represent downregulated proteins and the grey dots represent non-differentially phosphorylated proteins. (B) Most enriched KEGG pathways of the 18 differentially phosphorylated proteins. (C) PPI network of the 18 differentially phosphorylated proteins. (D) Western blot analysis of phosphorylation levels of AKT and its interacting proteins in ML-2 cells after treatment with increasing concentrations of CCM for 6 h. CCM, curcumin; KEGG, Kyoto Encyclopedia of Genes and Genomes; PPI, protein-protein interaction.

However, SC-79 produced the opposite results. Thus, the effects of curcumin were enhanced by afuresertib but attenuated by SC-79.

Curcumin promotes AML cell apoptosis by inactivating AKT. Annexin V and PI labeling followed by flow cytometry were used to detect apoptotic cells. The results demonstrated that both curcumin and afuresertib promoted apoptosis, while SC-79 suppressed apoptosis. Moreover, curcumin-induced apoptosis was stimulated by afuresertib, but diminished by SC-79 (Fig. 3A and B).

To identify the proteins involved in curcumin-induced apoptosis, the expression of three apoptosis-related proteins, including Bcl-2, caspase-3 and PARP, were examined. The results indicated that curcumin treatment decreased the antiapoptotic $\mathrm{Bcl}-2$ protein expression but increased the cleavage of caspase-3 (C-Casp3) and PARP (C-PARP) (Fig. 3C and D). Furthermore, the influence of curcumin on these three proteins could be enhanced by afuresertib, but was abrogated by SC-79.

The above results suggested that curcumin promoted AML cell arrest and apoptosis by inactivating AKT. However, $\mathrm{IC}_{50}$ values of curcumin were very weakly correlated with the levels of phosphorylated AKT in AML cell lines (Fig. S1). Thus suggested that curcumin also exerted antitumor roles via other pathways, besides the AKT pathway.

Curcumin and afuresertib synergistically reduce the leukemia burden in an AML xenograft mouse model. Next, the in vivo efficacy of curcumin and afuresertib for the treatment of 
A

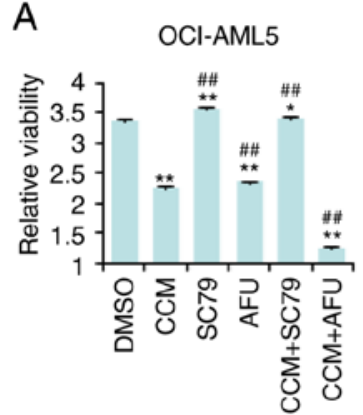

$\mathrm{B}$

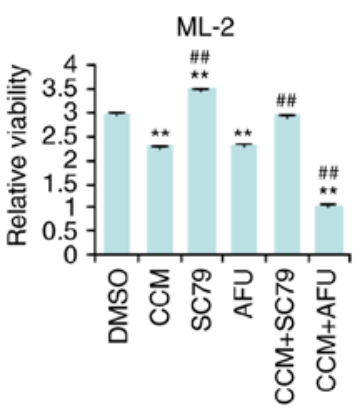

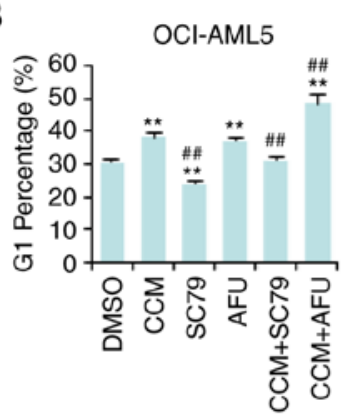

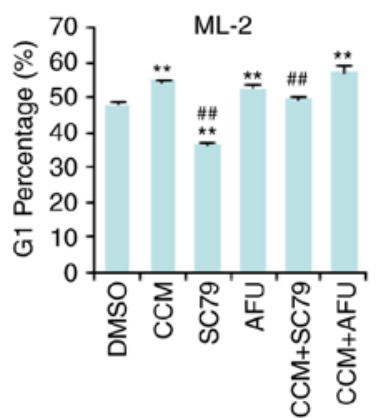

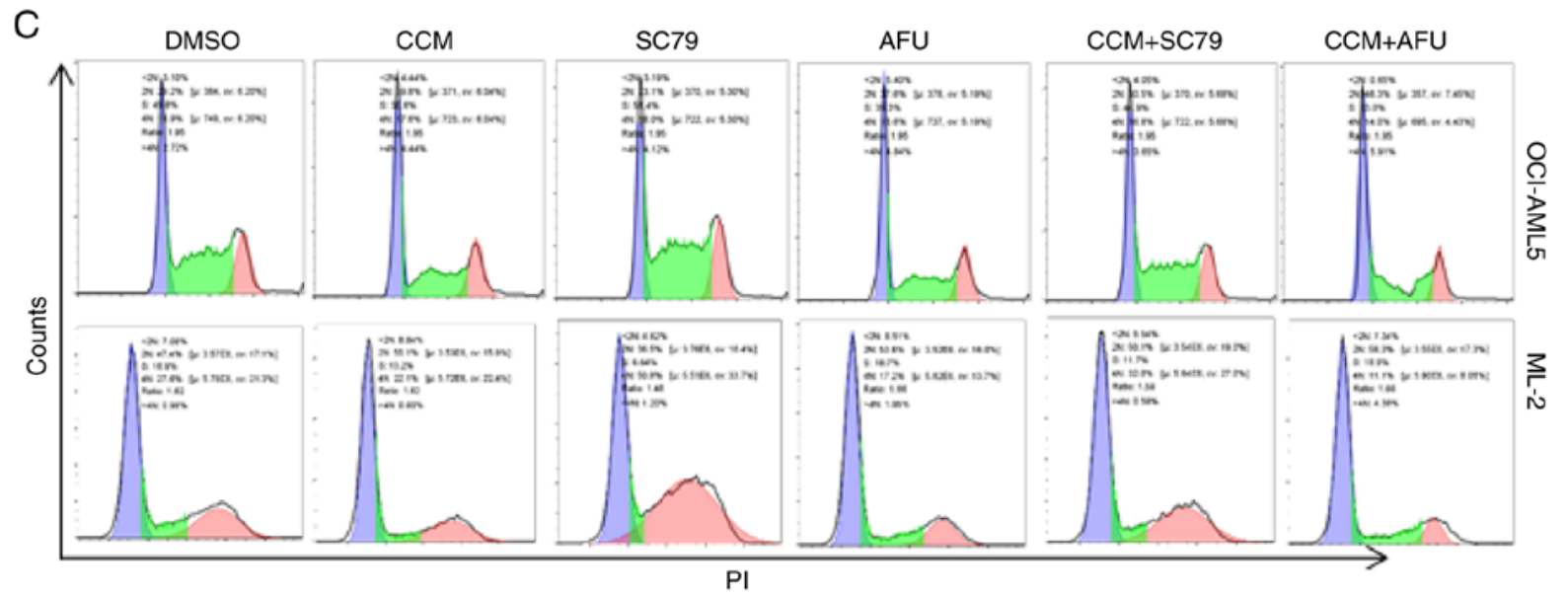
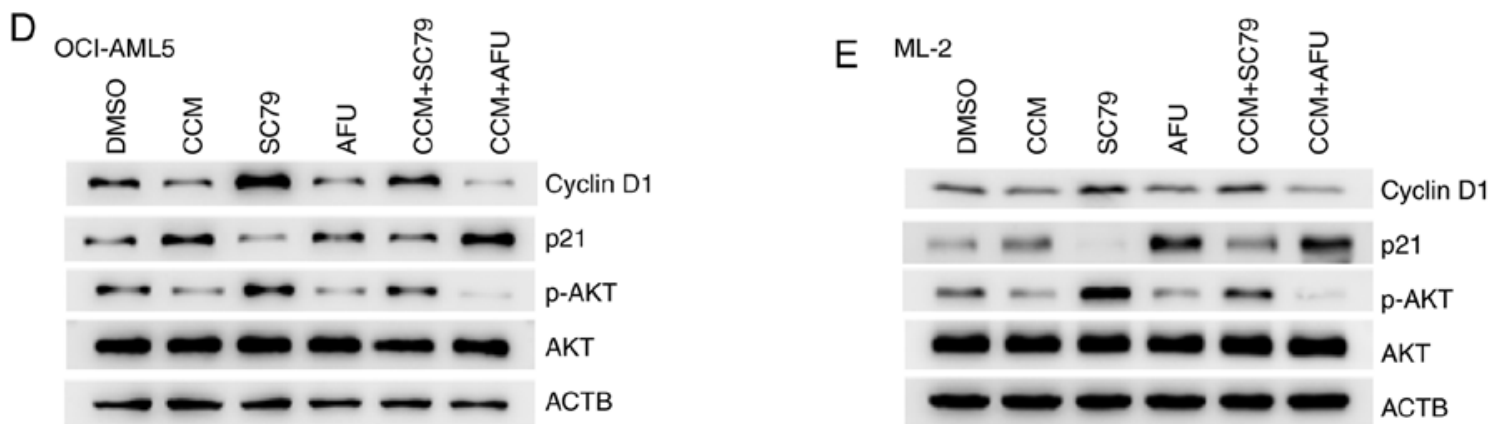

Figure 2. Curcumin (CCM) suppresses AML cell proliferation by inactivating AKT. OCI-AML5 and ML-2 cells were treated with CCM (25 $\mu$ M), SC-79 $(10 \mu \mathrm{M})$ or AFU $(10 \mu \mathrm{M})$ alone or in combination for $24 \mathrm{~h}$. After treatment, MTT assay, flow cytometry and western blotting were performed. (A) Cell viability results from triplicate experiments. (B) Percentage of cells in the G1 phase. Data are presented as the mean \pm SD of triplicate experiments. (C) Representative images of flow cytometry. (D and E) Expression levels of cell cycle-related proteins were detected via western blotting in OCI-AML5 and ML-2 cells. * $\leq 0.05$ and ${ }^{* *} \mathrm{P} \leq 0.01$ vs. DMSO; ${ }^{\# \#} \mathrm{P} \leq 0.01$ vs. CCM. AFU, afuresertib; CCM, curcumin; AML, acute myeloid leukemia.

AML was evaluated. NOD/SCID mice were intravenously injected with $1 \times 10^{6} \mathrm{ML}-2$ cells. Drug treatment began 15 days after injection and continued every other day for 16 days. After treatment, peripheral blood mononuclear cells (PBMCs) and bone marrow mononuclear cells (BMMCs) were isolated and evaluated for human hematopoietic (hCD45) chimerism via flow cytometry (Fig. 4). Compared with the control group (VEH), the mice treated with curcumin (CCM) or afuresertib (AFU) either alone or in combination (CCM+AFU) had fewer human $\mathrm{CD}^{4} 5^{+}$cells in the bone marrow and peripheral blood. Moreover, combination drug therapy was more effective than single drug therapy in reducing the chimerism of hCD45 (Fig. 4). These results indicated that curcumin and afuresertib synergistically suppressed the engraftment of AML cells.

The mice treated with curcumin and afuresertib either alone or in combination had a smaller and lighter spleen compared with the control mice (Fig. 5A and B). Thus, it was suggested that treatment with curcumin or afuresertib could decrease splenomegaly in AML mice. IHC using an anti-hCD45 antibody demonstrated that, compared with control mice, the mice treated with curcumin or afuresertib had decreased dissemination of AML cells in the spleen, and the combinational use of curcumin and afuresertib was more effective compared with the use of a single drug (Fig. 5C).

Subsequently, Ki-67 staining and TUNEL assay were conducted to evaluate cell proliferation and apoptosis, respectively. The results (Fig. 5C) demonstrated that treatment with curcumin or afuresertib significantly increased apoptosis but decreased AKT phosphorylation and the cell proliferation rate in spleen, while treatment with both drugs had stronger effects compared with treatment with a single drug. These findings suggested that treatment with curcumin or afuresertib suppressed the engraftment, proliferation and survival of AML cells, and that combination therapy had increased efficacy compared with monotherapy. 
A

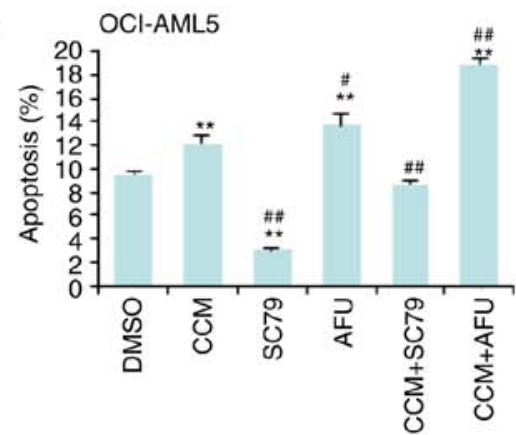

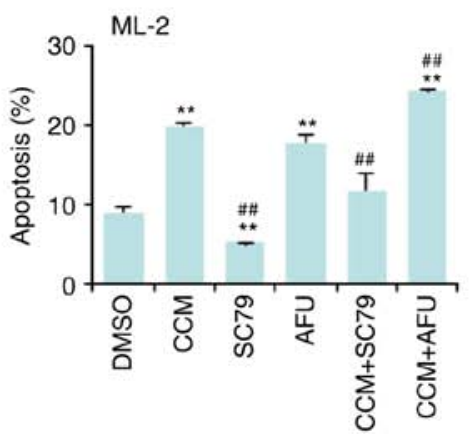

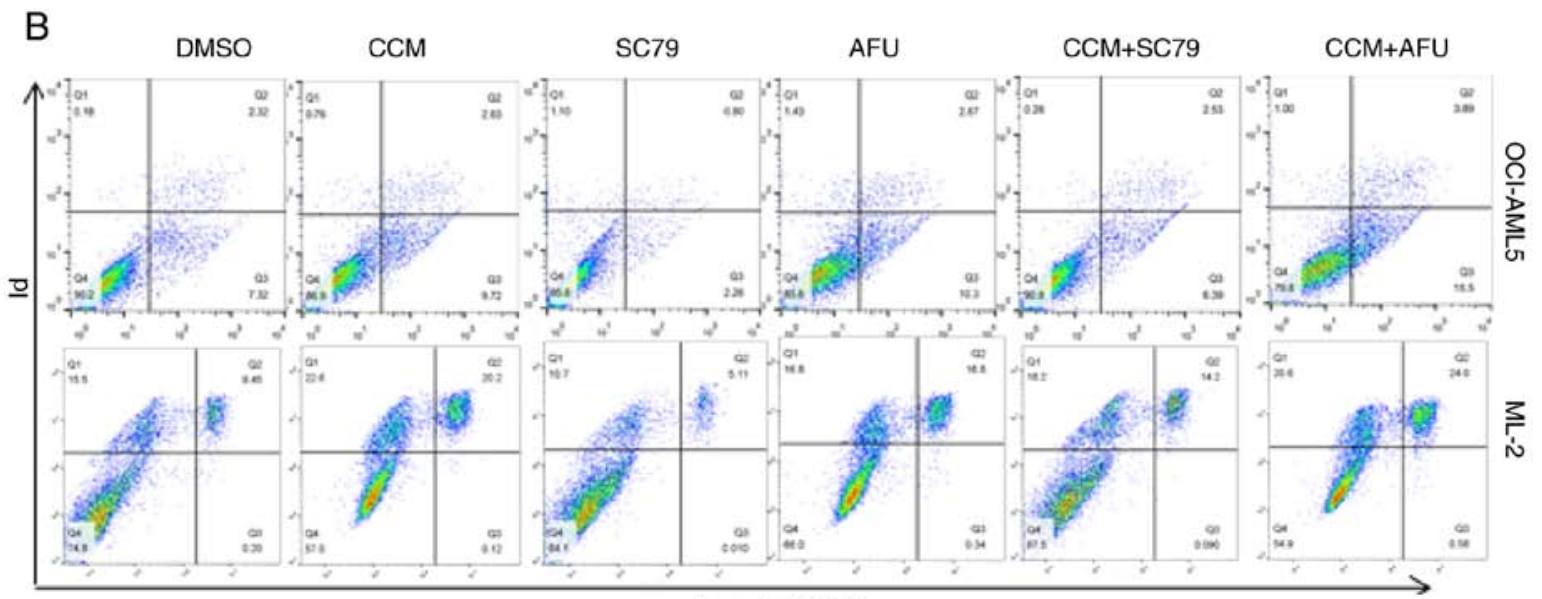

Annexin V-FITC
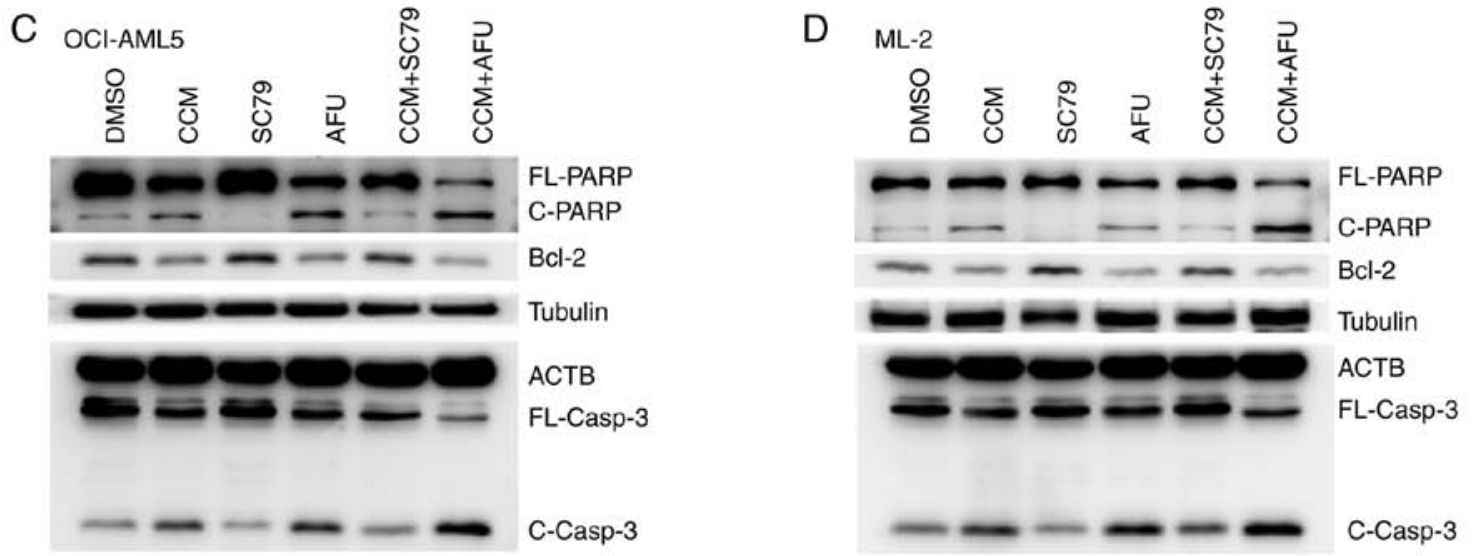

Figure 3. Curcumin (CCM) promotes AML cell apoptosis by inactivating AKT. ML-2 and OCI-AML5 cells were treated with CCM (25 $\mu \mathrm{M})$, SC-79 (10 $\mu \mathrm{M})$ or AFU $(10 \mu \mathrm{M})$ alone or in combination for $24 \mathrm{~h}$. After treatment, flow cytometry and western blotting were performed. (A) Percentage of apoptosis is presented as the mean $\pm \mathrm{SD}$ of triplicate experiments. (B) Representative images of flow cytometry. (C and D) Western blot analysis of FL-PARP, C-PARP, FL-casp3 and C-Casp3. ${ }^{* *} \mathrm{P} \leq 0.01$ vs. DMSO; ${ }^{\#} \mathrm{P} \leq 0.05$ and ${ }^{\# \#} \mathrm{P} \leq 0.01$ vs. CCM. FL-PARP, full length PARP; AFU, afuresertib; CCM, curcumin; AML, acute myeloid leukemia; C-PARP, cleaved-PARP; FL-casp3, full length caspase-3; C-Casp3, cleaved caspase-3; PARP, poly(ADP-ribose) polymerase.

\section{Discussion}

The present study compared the cytotoxicity of four phytochemicals (curcumin, EGCG, genistein and resveratrol) and identified that curcumin had the strongest anti-acute myeloid leukemia (AML) efficacy. It has been reported that curcumin has multiple targets and exerts its role via different molecular mechanism in various cancer types (11). Recently, several studies have revealed that curcumin can inhibit the phosphorylation of certain kinases, such as AKT (12), JAK1 (13) and p38MAPK (14). To identifying the targets of curcumin in AML, the present study performed a phosphorylation antibody array to detect the influence of curcumin on 55 phosphorylated proteins in five signaling pathways (MAPK, AKT, JAK/STAT, NF- $\kappa B$ and TGF- $\beta$ ). The present results suggested that curcumin decreased the phosphorylation levels of 14 proteins but increased the phosphorylation levels of four proteins. Then, a protein-protein interaction (PPI) network of these 18 proteins was conducted, in which AKT was a hub, indicating that AKT was a main target of curcumin. 
A PBMCs

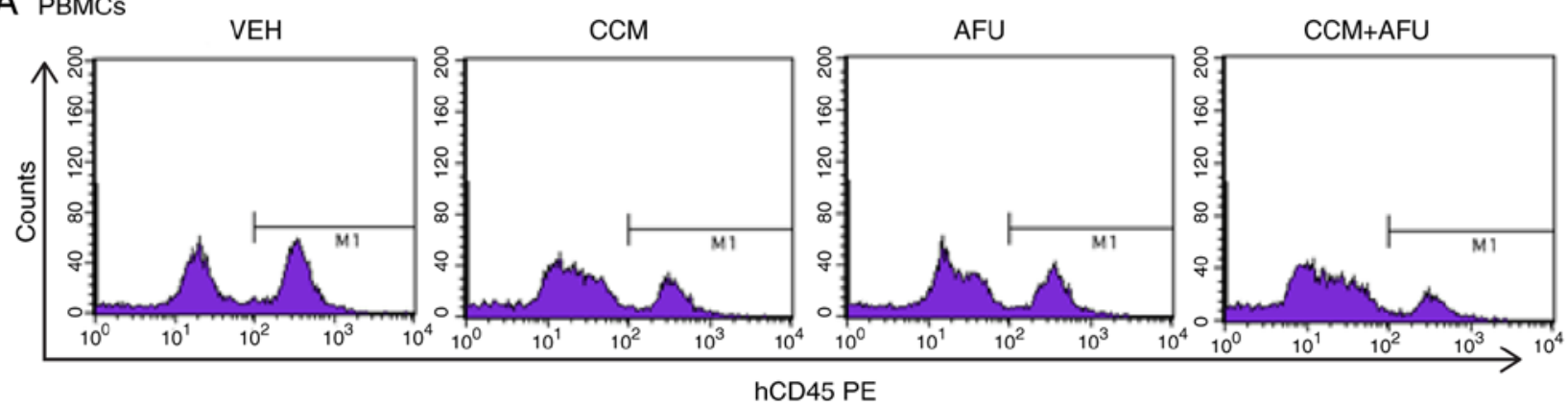

B BMmCs
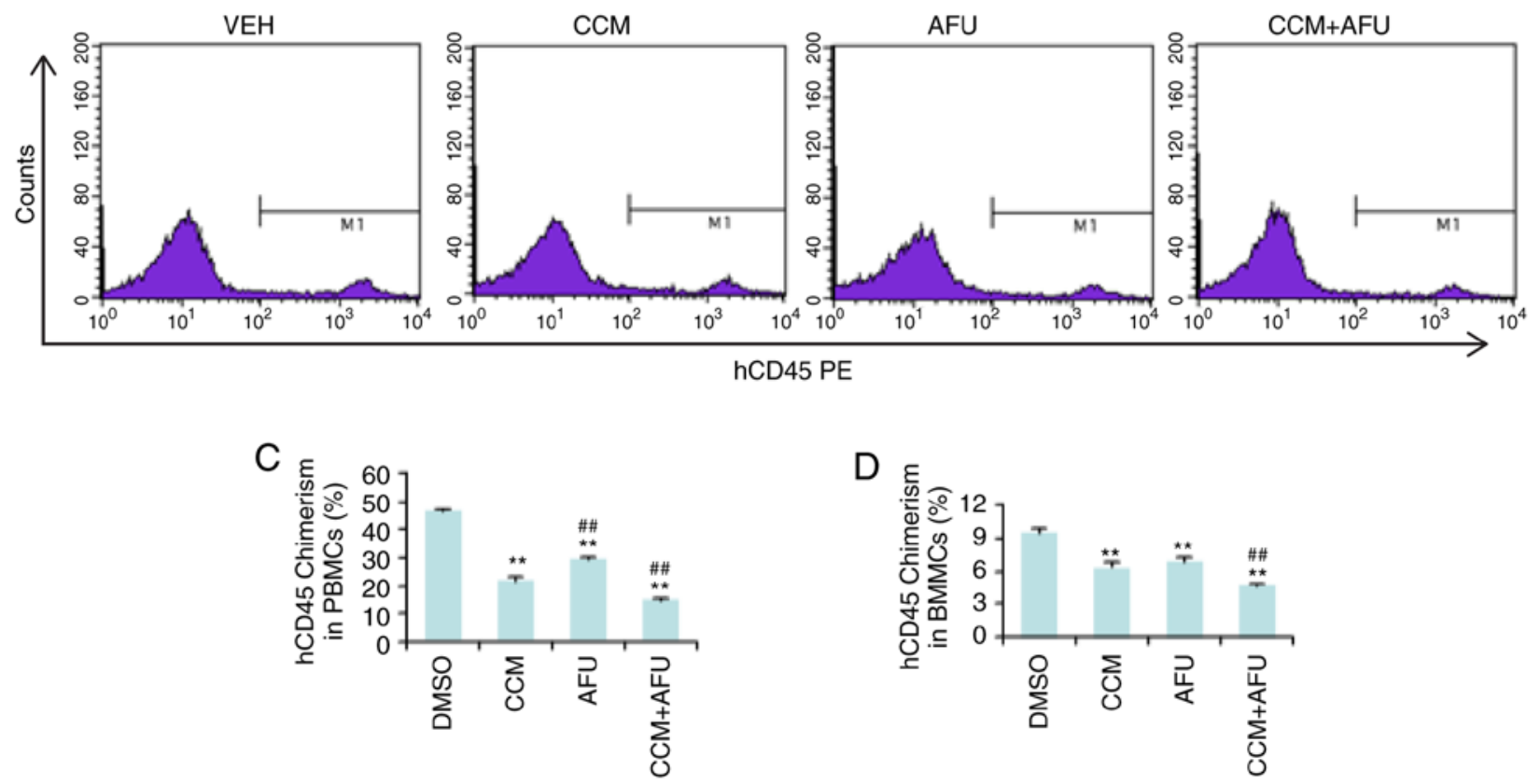

Figure 4. Curcumin (CCM) and afuresertib (AFU) synergistically inhibit engraftment of AML cells in PB and BM of mice. NOD/SCID mice were pretreated with intraperitoneal injection of $20 \mathrm{mg} / \mathrm{kg}$ busulfan $24 \mathrm{~h}$ before inoculation, and were then injected intravenously with $1 \times 10^{6} \mathrm{ML}-2$ cells. At 15 days after inoculation, the mice were randomly divided into four groups (5 mice per group), and were treated with VEH, CCM, AFU or CCM+AFU via oral gavage every other day for 16 days. PBMCs and BMMCs were isolated and evaluated for human hematopoietic (hCD45) chimerism via flow cytometry. (A and B) Representative images from flow cytometry. $(\mathrm{C}$ and $\mathrm{D}) \mathrm{Data}$ are presented as the mean $\pm \mathrm{SD}$ of three mice. ${ }^{* *} \mathrm{P} \leq 0.01 \mathrm{vs}$. VEH; ${ }^{* \prime} \mathrm{P} \leq 0.01 \mathrm{vs}$. CCM. $\mathrm{PB}$, peripheral blood; BM, bone marrow; VEH, vehicle; PBMCs, peripheral blood mononuclear cells; BMMCs, bone marrow mononuclear cells; AFU, afuresertib; CCM, curcumin; AML, acute myeloid leukemia.

Protein kinase B (AKT/PKB) is frequently overactivated in $\mathrm{AML}$, and its phosphorylation is an independent poor prognostic factor of overall survival in adult de novo AML (23). $\mathrm{AKT}$ is a serine threonine kinase that contains three isoforms: AKT1, AKT2 and AKT3. It has been reported to serve roles in various cellular pathways, including proliferation, apoptosis and angiogenesis. Cyclin D1, which regulates the $\mathrm{G}_{1} / \mathrm{S}$ check point of the cell cycle, has been reported to be upregulated by the AKT/glycogen synthase kinase $3 \beta$ axis (24). However, p21, a negative regulator of the cell cycle $\mathrm{G}_{1} / \mathrm{S}$ transition, is negatively regulated by AKT (25). AKT also promotes leukemia $\mathrm{T}$ cells by enhancing the transcription of Bcl-2 (26). The present results suggested that curcumin treatment increased AKT phosphorylation and p21 expression but decreased the expression levels of cyclin D1 and Bcl-2 in AML cells. Moreover, the effects of curcumin on the expression levels of p21, cyclin D1 and Bcl-2 were enhanced by the AKT inhibitor but were suppressed by the AKT activator. Therefore, it was indicated that curcumin may function via AKT. However, the sensitivities to curcumin of AML cell lines were not significantly correlated with their levels of AKT phosphorylation, suggesting that curcumin still functioned via other pathways, besides tbe AKT pathway.

The present study demonstrated the anti-AML effect of curcumin both in vitro and in vivo, and this effect was increased by the combination with afuresertib. Afuresertib has been reported to exert antitumor effects in ovarian cancer (27), malignant pleural mesothelioma (28) and chronic lymphocytic leukemia (29). However, to the best of our knowledge, its role in AML has not been previously reported. The present study was the first report that afuresertib could potentially be used for the treatment of AML.

In conclusion, the present study demonstrated that curcumin decreased the survival and proliferation of AML cells in vitro, as well as AML cell proliferation in hematopoietic tissue and 
A

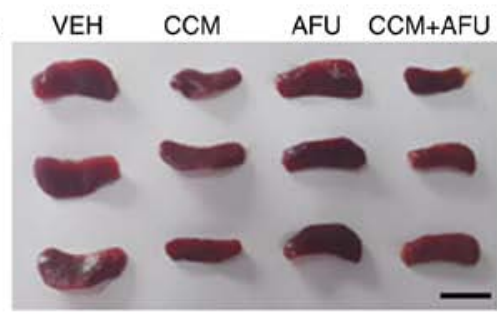

C
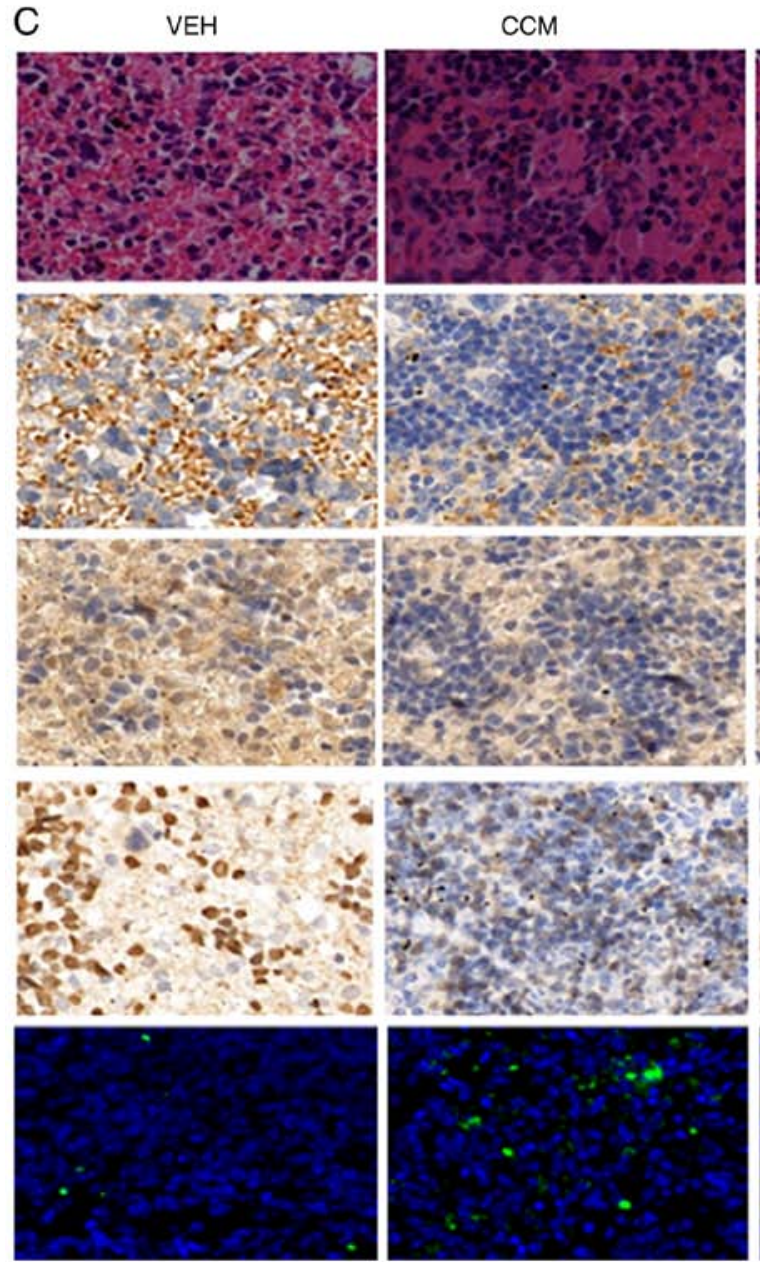

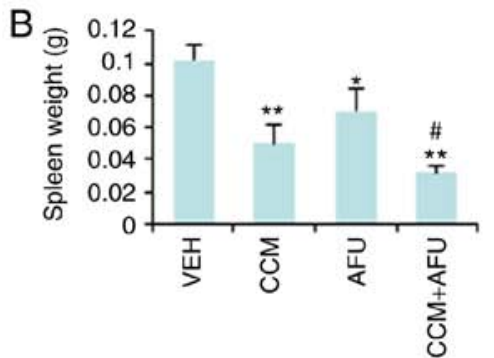

AFU
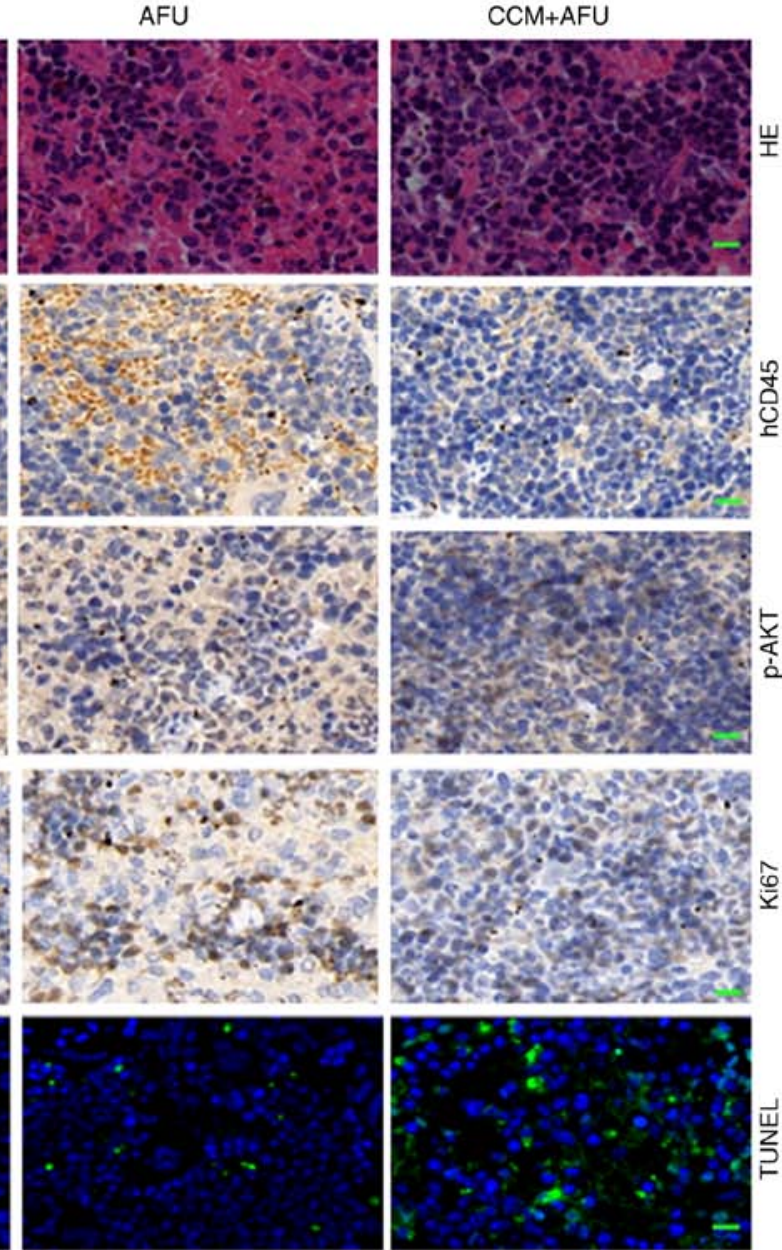

Figure 5. Curcumin (CCM) and afuresertib (AFU) synergistically inhibit engraftment, proliferation and survival of AML cells in the spleens of mice. NOD/SCID mice were pretreated with intraperitoneal injection of $20 \mathrm{mg} / \mathrm{kg}$ busulfan $24 \mathrm{~h}$ before inoculation and were then injected intravenously with $1 \times 10^{6} \mathrm{ML}-2$ cells. At 15 days after inoculation, the mice were randomly divided into four groups (5 mice per group), and were treated with $\mathrm{VEH}$, CCM, AFU or CCM+AFU via oral gavage every other day for 16 days. (A) Gross appearance of spleen. Scale bar, $1 \mathrm{~cm}$. (B) Average weight of spleen. (C) H\&E, IHC and TUNEL assays of the spleen. Scale bar, $50 \mu \mathrm{m}$. ${ }^{*} \mathrm{P} \leq 0.05$ and ${ }^{* *} \mathrm{P} \leq 0.01$ vs. VEH; ${ }^{*} \mathrm{P} \leq 0.05$ vs. CCM. VEH, vehicle; AFU, afuresertib; CCM, curcumin; AML, acute myeloid leukemia; H\&E, hematoxylin and eosin; IHC, immunohistochemistry.

dissemination into non-hematopoietic tissues. Mechanistically, curcumin treatment suppressed AKT activation, leading to cell cycle arrest and apoptosis.

\section{Acknowledgements}

We would like to thank RayBiotech Inc. (Guangzhou, China) for the assistance in the phosphorylation array analysis.

\section{Funding}

This study was supported by the National Natural Science Foundation of China (grant nos. 81872256 and 82070155) and Hunan Key Laboratory of Pharmacodynamics and Safety Evaluation of New Drugs.

\section{Availability of data and materials}

All data generated and/or analyzed during the study are available from the corresponding author on reasonable request.

\section{Authors' contributions}

$\mathrm{CZ}$ and $\mathrm{XD}$ conceived and designed the study. HZ, YN and GZ performed the experiments. HZ, YN, CZ and XD analyzed 
and interpreted the data. $\mathrm{HZ}, \mathrm{CZ}$ and $\mathrm{XD}$ wrote the manuscript. All authors read and approved the manuscript and agree to be accountable for all aspects of the research in ensuring that the accuracy or integrity of any part of the work are appropriately investigated and resolved.

\section{Ethics approval and consent to participate}

All animal experiments were approved by the Animal Ethics Committee of Hunan Normal University and performed according to institutional animal care guidelines (no. 2018-037).

\section{Patient consent for publication}

Not applicable.

\section{Competing interests}

The authors declare that they have no competing interests.

\section{References}

1. Döhner H, Weisdorf DJ and Bloomfield CD: Acute myeloid leukemia. N Engl J Med 373: 1136-1152, 2015.

2. Tallman MS, Wang ES, Altman JK, Appelbaum FR, Bhatt VR, Bixby D, Coutre SE, De Lima M, Fathi AT, Fiorella M, et al: Acute myeloid leukemia, version 3.2019, NCCN clinical practice guidelines in oncology. J Natl Compr Cancer Netw 17: 721-749, 2019.

3. Oran B and Weisdorf DJ: Survival for older patients with acute myeloid leukemia: A population-based study. Haematologica 97: 1916-1924, 2012

4. Sami SA, Darwish NHE, Barile ANM and Mousa SA: Current and future molecular targets for acute myeloid leukemia therapy. Curr Treat Options Oncol 21: 3, 2020.

5. Wang XJ, Chen JY, Fu LQ and Yan MJ: Recent advances in natural therapeutic approaches for the treatment of cancer. J Chemother 32: 53-65, 2020.

6. Kian MM, Salemi M, Bahadoran M, Haghi A, Dashti N, Mohammadi S, Rostami S, Chahardouli B, Babakhani D and Nikbakht M: Curcumin combined with thalidomide reduces expression of STAT3 and Bcl-xL, leading to apoptosis in acute myeloid leukemia cell lines. Drug Des Devel Ther 14: 185-194, 2020.

7. Liang K, Bae KH, Nambu A, Dutta B, Chung JE, Osato M and Kurisawa M: A two-pronged anti-leukemic agent based on a hyaluronic acid-green tea catechin conjugate for inducing targeted cell death and terminal differentiation. Biomater Sci 8: 497-505, 2019

8. de Blas E, Estañ MC, Del Carmen Gomez de Frutos M, Ramos J, Del Carmen Boyano-Adánez M and Aller P: Selected polyphenols potentiate the apoptotic efficacy of glycolytic inhibitors in human acute myeloid leukemia cell lines. Regulation by protein kinase activities. Cancer Cell Int 16: 70, 2016.

9. Li Y, Guo Y, Feng Z, Bergan R, Li B, Qin Y, Zhao L, Zhang Z and Shi M: Involvement of the PI3K/Akt/Nrf2 signaling pathway in resveratrol-mediated reversal of drug resistance in HL-60/ADR cells. Nutr Cancer 71: 1007-1018, 2019.

10. Giordano A and Tommonaro G: Curcumin and cancer. Nutrients 11:2376, 2019.

11. Liczbinski P, Michałowicz J and Bukowska B: Molecular mechanism of curcumin action in signaling pathways: Review of the latest research. Phytother Res 34: 1992-2005, 2020.

12. Borges GA, Elias ST, Amorim B, de Lima CL, Coletta RD, Castilho RM, Squarize $\mathrm{CH}$ and Guerra EN: Curcumin downregulates the PI3K-AKT-mTOR pathway and inhibits growth and progression in head and neck cancer cells. Phytother Res 34 $3311-3324,2020$
13. Li Y, Sun W, Han N, Zou Y and Yin D: Curcumin inhibits proliferation, migration, invasion and promotes apoptosis of retinoblastoma cell lines through modulation of miR-99a and JAK/STAT pathway. BMC Cancer 18: 1230, 2018.

14. Hosseini A, Rasmi Y, Rahbarghazi R, Aramwit P, Daeihassani B and Saboory E: Curcumin modulates the angiogenic potential of human endothelial cells via FAK/P-38 MAPK signaling pathway. Gene 688: 7-12, 2019.

15. Zhou J, Duan H, Xie Y, Ning Y, Zhang X, Hui N, Wang C, Zhang J and Zhou J: MiR-193a-5p targets the coding region of AP-2alpha mRNA and induces cisplatin resistance in bladder cancers. J Cancer 7: 1740-1746, 2016.

16. Kouhpeikar H, Butler AE, Bamian F, Barreto GE, Majeed M and Sahebkar A: Curcumin as a therapeutic agent in leukemia. J Cell Physiol 234: 12404-12414, 2019.

17. Zhou C, Zhao XM, Li XF, Wang C, Zhang XT, Liu XZ, Ding XF, Xiang SL and Zhang J: Curcumin inhibits AP-2 $\gamma$-induced apoptosis in the human malignant testicular germ cells in vitro. Acta Pharmacol Sin 34: 1192-1200, 2013.

18. Yang L, Qiu J, Xiao Y, Hu X, Liu Q, Chen L, Huang W, Li X, Li L, Zhang J, et al: AP-2ßinhibits hepatocellular carcinoma invasion and metastasis through Slug and Snail to suppress epithelial-mesenchymal transition. Theranostics 8: 3707-3721, 2018.

19. Carlos-Reyes A,Lopez-GonzalezJS,Meneses-Flores M,GallardoRincón D, Ruíz-García E, Marchat LA, Astudillo-de la Vega H, de la Cruz ON and López-Camarillo C: Dietary compounds as epigenetic modulating agents in cancer. Front Genet 10: 79, 2019.

20. He X and Zhang J: Why do hubs tend to be essential in protein networks? PLoS Genet 2: e88, 2006

21. Masamha CP and Benbrook DM: Cyclin D1 degradation is sufficient to induce G1 cell cycle arrest despite constitutive expression of cyclin E2 in ovarian cancer cells. Cancer Res 69: 6565-6572, 2009.

22. Georgakilas AG, Martin OA and Bonner WM: p21: A two-faced genome guardian. Trends Mol Med 23: 310-319, 2017.

23. Prijic S, Ugrina I, Labar B, Nemet D, Batinić J, Zadro R, Ries S, Gjadrov-Kuvedžić K, Davidović S and Batinić D: Prognostic significance of constitutive phosphatidylinositol 3-kinase/akt and mitogen-activated protein kinase phosphorylation in acute myeloid leukemia. Leuk Lymphoma 56: 2281-2288, 2015.

24. Qin Z, Li Y, Li Y and Liu G: Tumor necrosis factor alpha stimulates proliferation of dental pulp stem cells via akt/glycogen synthase kinase-3ß/cyclin D1 signaling pathway. J Endod 41: 1066-1072, 2015.

25. Hu Z, Long T, Ma Y, Zhu J, Gao L, Zhong Y, Wang X, Wang X and Li Z: Downregulation of GLYR1 contributes to microsatellite instability colorectal cancer by targeting p21 via the p38MAPK and PI3K/AKT pathways. J Exp Clin Cancer Res 39: 76, 2020.

26. Wan YJ, Yang Y, Leng QL, Lan B, Jia HY, Liu YH, Zhang CZ and Cao Y: Vavl increases bcl-2 expression by selective activation of rac2-akt in leukemia T cells. Cell Signal 26: 2202-2209, 2014.

27. Blagden SP, Hamilton AL, Mileshkin L, Wong S, Michael A, Hall M, Goh JC, Lisyanskaya AS, DeSilvio M, Frangou E, et al: Phase IB dose escalation and expansion study of AKT inhibitor afuresertib with carboplatin and paclitaxel in recurrent platinum-resistant ovarian cancer. Clin Cancer Res 25: 1472-1478, 2019.

28. Yamaji M, Ota A, Wahiduzzaman M, Karnan S, Hyodo T, Konishi H, Tsuzuki S, Hosokawa Y and Haniuda M: Novel ATP-competitive Akt inhibitor afuresertib suppresses the proliferation of malignant pleural mesothelioma cells. Cancer Med 6: 2646-2659, 2017.

29. Chen CI, Paul H, Le LW, Wei EN, Snitzler S, Wang T, Levina O, Kakar S, Lau A, Queau M, et al: A phase 2 study of ofatumumab $\left(\right.$ Arzerra ${ }^{\circledR}$ ) in combination with a pan-AKT inhibitor (afuresertib) in previously treated patients with chronic lymphocytic leukemia (CLL). Leuk Lymphoma 60: 92-100, 2019.

This work is licensed under a Creative Commons Attribution-NonCommercial-NoDerivatives 4.0 International (CC BY-NC-ND 4.0) License. 